\title{
Changes in Lipid Profiles, Insulin Resistance and Serum Fibroblast Growth Factor 21 Following Three Types of Exercise Training in Obese Children
}

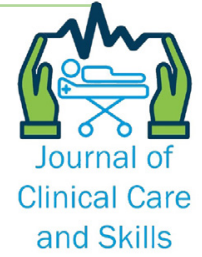

\section{ARTICLE INFO}

\section{Article Type}

Original Research

\section{Authors}

Mohammadi J. ${ }^{1} P h D$,

Ramezani A. ${ }^{2} P h D$,

Hosseini M. ${ }^{3} P h D$

MohammadiB. ${ }^{* 4} M D$

Gaeini A. $^{5} \mathrm{PhD}$

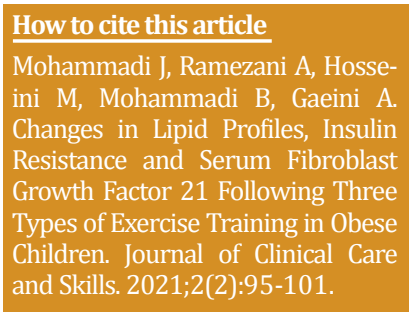

${ }^{1}$ Department of Physiology, Medicine Faculty, Yasuj University of Medical Sciences, Yasuj, Iran

${ }^{2}$ Department of Sports Physiology, Faculty of Sport Sciences, Shahid Rajaee Teacher Training University, Tehran, Iran ${ }^{3}$ Department of Sports Cardiovascular and Respiratory Physiology, Faculty of Sport Sciences, Shahid Rajaee Teacher Training University, Tehran, Iran

${ }^{4}$ Department of Pediatrics, Medicine Faculty, Yasuj University of Medical Sciences, Yasuj, Iran

${ }^{5}$ Department of Sports Physiology, Faculty of Sport Sciences, Tehran University, Tehran, Iran

\section{*Correspondence}

Address: Department of Pediatrics, Medicine Faculty, Yasuj University of Medical Sciences, Yasuj, Iran. Postal code: 7591994799.

Phone: +98 (910) 7040572

Fax: +98 (74) 33230290

drmohammadi114@gmail.com

\section{Article History}

Received: January 21, 2021

Accepted: May 02, 2021

ePublished: June 20, 2021

\section{A B S T R A C T}

Aims Obesity and immobility predispose to cardiovascular disease. Today, changing childhood lifestyle may affect cardiovascular disease factors. This research aimed to determine the effect of three types of exercise training, including endurance exercise, resistance exercise, and combined exercise, on lipid profiles, insulin resistance, and serum FGF21 in obese children. Materials \& Methods This experimental study was conducted on a total of 8-12 years old male students of elementary schools in Yasuj, Iran ( $N=18038)$ in October 2006. Sixty obese students, by the simple and voluntarily sampling method, were randomly allocated four groups (15 individuals): Resistance exercise, endurance exercise, and combined exercise, and control. Blood sampling was taken 24 hours before the start of the training period and 48 hours after the end latest session of the training period at the Laboratory of Shahid Beheshti Hospital in Yasuj, and the factors such as Glucose, TC, TG, LDL, VLDL, HDL, VO2max, HOMA-Insulin Resistance, and Serum FGF-21 were measured. Data were analyzed by SPSS 21 software using the ANCOVA test.

Findings Their BMI and VO2max were 32.36 ? $2.31 \mathrm{~kg} / \mathrm{m} 2$ and 23.06 ? $1.72 \mathrm{lit} / \mathrm{kg} / \mathrm{min}$, respectively, which showed that subjects were non-active (sedentary) obese in pre-training. Results showed that eight weeks of EE, RE, and CE had a significant effect on increasing serum HDL $(F=3.04 ; p<0.001)$ and on decreasing of glucose $(F=2.98 ; p<0.001), T C(F=4.33$; $\mathrm{p}<0.001)$, TG $(\mathrm{F}=5.44 ; \mathrm{p}<0.001)$, LDL $(\mathrm{F}=4.86 ; \mathrm{p}<0.001)$, VLDL $(\mathrm{F}=5.58 ; \mathrm{p}<0.001)$. There was a significant difference between the training intervention groups in pre-training and posttraining in the HOMA-Insulin Resistance variable $(\mathrm{F}=3.28$; $\mathrm{p}<0.001)$, which had decreased, and serum FGF-21 ( $F=2.45$; $\mathrm{p}<0.001)$ which had increased. Also, results illustrated that the EE method was better than RE and CE in improving these variables.

Conclusion Applied training methods in this study, especially endurance exercise, can be considered suitable interventions to reduce cardiovascular risk factors related to obesity in childhood.

Keywords Exercise; Lipid; Insulin resistance; Fibroblast growth factor 21

\section{I T A T I O N L I N KS}

[1] Effect of training in water on serum adiponectin ... [2] Heart disease and stroke statistics ... [3] Associations between meeting combinations ... [4] Effects of endurance training on apoB. apoA1 ... [5] The relationship between leptin level ... [6] Ankle brachial index combined with Framingham ... [7] The long-term effects of resistance training ... [8] Comparison of the effect of Ramazan month's ... [9] Laboratory assessment of cardiometabolic ... [10] The prevalence of coronary artery risk factors ... [11] effect of endurance training on skeletal muscle ... [12] Acute exercise induces FGF21 expression ... [13] Effect of endurance, strength and combined ... [14] Changes in body weight, C-reactive protein ... [15] Levels of circulating selenoprotein P ... [16] Cardiorespiratory fitness and ... [17] Serum adiponectin levels in people ... [18] Resistance versus endurance training ... [19] Laboratory method for determining ... [20] A new direction in psychology and health ... [21] Effects of endurance and circuit ... [22] Survey on metabolic cardiovascular risk ... [23] A cross-sectional and prospective ... [24] The positive effects of exercise in ... [25] Improved Insulin resistance, adiponectin ... [26] ACSM's new preparticipation health ... [27] Age-predicted maximal heart rate ... [28] Effects of three methods of exercise ... [29] Overweight and obesity in children ... [30] Relationship between physical activity ... [31] Association of cardiovascular ... [32] Resistance exercise in individuals with ... [33] Exercise increase adiponectin levels and ... [34] Strength training increases in insulin ... [35] Skeletal muscle insulin resistance and ... [36] Increasing insulin resistance accentuates... [37] Asystematic review and meta-analysis ... [38] Effects of a three-month combined exercise ... 


\section{Introduction}

Cardiovascular diseases are increasing, particularly in low-and middle-income countries [1-3]. The consequences of obesity are atherosclerosis, hypertension, and type II diabetes. About $22 \%$ of the global population had overweight, and $8.8 \%$ was obese [4]. In addition, overweight and obesity is the most common health problem among Iranian children and teenagers [1]. Today, at least $27 \%$ of children are obese. To the prevalence in children and adolescents, it is estimated that $70 \%$ of obese children become obese adults [1]. Research shows, lack of exercise can cause obesity and some cardiovascular diseases that can increase the factors threatening the lives of people in the future $[5,6]$. Besides, in Iran, about ninety thousand deaths are associated with cardiovascular disease ${ }^{[4]}$.

Studies show that overweight and obesity, age, smoking, drugs, alcohol, and physical activity reduction can increase cardiovascular risk factors. These factors include low-density lipoprotein cholesterol (LDL), high-density lipoprotein cholesterol (HDL), Triglycerides (TG), Total Cholesterol (TC), and blood pressure [7, 8]. However, the research shows that other factors have been known as risk factors, such as fibroblast growth factor 21 (FGF21), which may also have insulin resistance and cardiovascular health risks, thus threatening people's lives $[7,8]$. FGF21 is a protein that in mammals is encoded by the FGF21 gene [1]. FGF21 is expressed in many tissues, containing liver, white and brown adipose tissue, and pancreas [8, 9]. FGF21 plays a role in regulating lipid and glucose metabolism, related to obesity metabolic problems ${ }^{[9]}$. Research showed concentrations of FGF21 increases in both children and adults obese. FGF21 serum and serum levels are associated with body mass index and adiponectin. Recent findings in children showed that FGF21 has a direct relationship with triglycerides and insulin resistance [9]. Many studies have shown that lower LDL levels, TG, and Tc are associated with high fitness levels [7, 8]. Endurance exercise includes a wide range of sporting events such as boating (about 3 minutes) to two marathons (about 2-3 hours) and even some cycling (about 4 hours). Although the duration of these events varies, the sources of ATP production in them are often provided by the aerobic system. Resistance exercise includes some different techniques (for example, weight and strength exercise). However, resistance exercise usually involves sports activities performed against resistance to increase strength and endurance. Although the type and intensity of resistors can vary, the sources of ATP production in them are often provided by the anaerobic system. Compound exercise includes a combination of endurance and resistance exercises $[8,9]$.

Some studies have expressed that different exercises did not significantly change cardiovascular risk factors [9-12]. Most of them examined the effects of exercise on cardiovascular risk factors that were lasted longer than 8-week courses [9-12]. In another study, the effect of 12 weeks of endurance training on blood lipid profile values and body fat\% in obese 16 non -athlete's students aged between 20 and 25 years were studied. Therefore, identifying the biochemical factors in cardiovascular diseases' development process is of significant importance [1317]. All previous findings confirmed that week 12 training (done three times a week) improves the condition and reduces risk factors in the experimental group compared with the control one [13]. In another study, Kim and colleagues examined the effect of twelve weeks of regular endurance training on the rate of blood lipids profile, insulin resistance teenagers in students with an average age of 17, and their body mass index higher than 25 [18]. They found that 12 weeks of regular endurance training five times a week, each session lasting 50 minutes (10 minutes warm-up, 35 minutes of endurance training, and a 5-minute cool-down), reduces blood fat levels and insulin resistance in these people [15].

According to the above, different training methods such as endurance, resistance and combination can affect traditional and new cardiovascular risk factors such as lipid profile, blood sugar, serum insulin, and serum FGF21. In some cases, the mentioned research has reached contradictory results, requiring more research in this field. On the other hand, most studies have used long-term training courses (over eight weeks) that require more time and expenditure for the person and the possibility that the person cannot tolerate and continue to run a long-term program psychologically. Also, no such research was found in obese children. So based on what has stated, the researchers are seeking to answer whether, after eight weeks of endurance training, resistance, and combined exercise training, cardiovascular risk factors, including a blood lipid profile, insulin resistance, and FGF21 in obese children?

Moreover, if so, what exercise training can make the most differences? According to the above, different training methods such as endurance, resistance and combination can affect traditional and new cardiovascular risk factors such as lipid profile, blood sugar, serum insulin, and serum FGF21. In some cases, the mentioned research has reached contradictory results, requiring more research in this field. On the other hand, most studies have used longterm training courses (over eight weeks) that require more time and expenditure for the person and the possibility that the person can not tolerate and continue to run a long-term program psychologically. Also, no such research was found in obese children. So based on what has stated, the researchers seek to answer whether, after eight weeks of endurance training, resistance and combined exercise training, 
cardiovascular risk factors, including a blood lipid profile, insulin resistance, and FGF21 in obese children? Moreover, if so, what exercise training can make the most differences? Therefore, this study aimed to determine the effect of three types of exercise training, including endurance exercise, resistance exercise, and combined exercise, on lipid profiles, insulin resistance, and serum FGF21 in obese children.

\section{Materials and Methods}

This experimental study was conducted on 8-12 years old male students of elementary schools in Yasuj (N=18038), Iran, in October 2006. Sixty obese students by simple and voluntarily sampling method were randomly allocated four groups (15 individuals): Resistance exercise (RE), endurance exercise (EE), and combined exercise (CE) that included endurance plus resistance exercise performed in different sessions during a week, and control (C). All boys' primary schools in BoyerAhmad were selected to find obese male students. Then, the following formula was used to calculate the sample size: $\varnothing=\sqrt{\frac{\mathrm{n} \delta^{2}}{\mathrm{ks}^{2}}}$

Wherein: $\delta$ : Minimum clinically acceptable effect, $\mathrm{s}^{2}$ : variance, $\mathrm{n}$ : The sample size is suggested for each group, $\mathrm{k}$ : The number of groups included in the study. Inclusion criteria including inactivity or did not participate in a regular exercise program during the last six months, and their Z-score was above +2 . The use of medications and nutritional supplements in the form of oral or nutritional supplements that in some way affect the body's metabolism or having a special diet and a history of chronic and metabolic diseases such as asthma, diabetes, metabolic syndrome, cardiovascular disease, etc. were exclusion criteria.

This study was approved by the Ethics Committee of Yasuj University of Medical Sciences. All parents of the participants were informed about the study's goal, and they who adopted to participate signed informed consent. All of the participants were taken in medical tests before being allowed in the study. At the end of the study, all children followed to receive medical care from the original service. Firstly, the body mass index (BMI) of participants was examined, and a physician was assessed their physical condition. The weight of participants was measured on a scale without shoes and excess clothing. Weight was measured by Omron scale Model HBF516 (Omron Healthcare, Inc, China). The height was measured in a standing position using a stadiometer Model 3PHTROD-WM (DETECTO, USA). BMI was obtained by dividing body weight $(\mathrm{kg})$ by the square of the height (m). Resting Heart Rate measured the mean \pm SD score of 3 times for each participant in the morning, and it was assessed by a stethoscope (HiTec, Canada) at-rest state. Maximal oxygen

Journal of Clinical Care and Skills consumption $\left(\mathrm{VO}_{2} \max \right)$ is the highest oxygen consumption rate in $1 \mathrm{~kg}$ of muscle tissue for one minute [5]. $\mathrm{VO}_{2}$ max was measured the Progressive Aerobic Cardiovascular Endurance Run (PACER) fitness test that was performed as follows: Sign a 20meter course with enough space for each person to run. Each person should run the full 20 -meters after the beep sound listened, arrive at the line at the end of the 20 meters, then wait for the CD to play deep sound. Once a person fails to reach the other end before a beep twice, the test was finished. The test results were entered into the Fitness Gram software to calculate the $\mathrm{VO}_{2}$ max. Reliability of the PACER fitness test has been demonstrated in children ages 616 years old [10], while validity was shown in children ages 8-19 years old [10,17]. The exercise protocol included an 8-week course of endurance, resistance, and combination exercises performed four times a week (in the morning) and were as follows. EET sessions contained 15 minutes' warm-up with slow running, stretching movements and then performed 4 to 7 sets of running with moderate intensity. Each set performed 5 minutes of aerobic running with 1minute rest between sets. Running intensity was between $\% 50$ to $\% 75$ of heart rate reserve computed with the Karvonen formula [5]. To apply the principle of overload, intensity rate and sets repetitions increased every two weeks. Finally, cool down was performed by stretching motions and slow running for ten minutes. Exercise intensity was calculated by the following formula: Exercise Heart Rate $=[(\mathrm{HRmax}-\mathrm{HRr}) \times$ selected intensity\% $]+\mathrm{HRr}$ (Karvonen formula). HRmax was calculated by this formula [208-0.7 (age)] [19]. HRr was calculated by the mean of 3 assessments in the morning. RE protocol included circuit exercise training (performed in 5 stations) with 1-minute rest between sets. Exercise training stations performed in 3 sets with eight repetitions and 1-minute rest between sets. Resistance training intensity was \%50-75 of $1 \mathrm{RM}$ (one repetition maximum). 1RM is the maximum weight that an individual would once lift was estimated by Brzycki's equation [15]: 1RM=dislocated weight $\quad(\mathrm{kg}) /[1.0278$-(repetition to exhaustion $\times 0.0278)]$. The RE protocol was aimed to strengthen the muscles and to improve cardiovascular endurance [3]. In the per session, the warm-up was done for 15 minutes, then RET program was done for 25-40 minutes, and about 10 minutes of cool-down were performed. RE program was as follows: knee extension with the machine for quadriceps muscle contraction, knee curl with the machine for hamstring muscle contraction, butterfly exercise with the machine for latissmus Dorsi contraction, triceps extension with dumbbells, and biceps curl with a dumbbell. The number of sets was three, and the repetitions were 6 or 8 repetitions up to 12 or 14 repetitions and with an intensity of 50 to $75 \%$ of a maximum repetition. Because researchers made the exercise protocols, they were approved by 
sports experts, and they were performed for a week as a pilot study. The results of the pilot study were confirmed by sports experts. Then, the exercise protocols were implemented. The CE protocol contained two sessions of RE and two sessions of EE. Group C did their daily work as usual. All of the exercise protocols were administered by sports physiologist's experts. The exercise training place was the Sports base Championship in Yasuj, Iran. Blood sampling was taken 24 hours before the start of the training period and 48 hours after the end latest session of the training period at the Laboratory of Shahid Beheshti Hospital in Yasuj. For the blood sampling, the subjects were not any intense activity two days before the test. Blood samples of $10 \mathrm{ml}$ were taken from the right brachial artery after 14 hours of fasting. The plasma samples were obtained after centrifuging was done at four ${ }^{\circ} \mathrm{C}$ with $3000 \mathrm{RPM}$ for 15 minutes. TC, HDL-C, and TG concentrations were measured in enzymatic ways using an automated clinical chemistry analyzer (Dimension RxL Max, Siemens Healthcare Diagnostics, and Germany). LDL$\mathrm{C}$ was measured by the Friedewald, Levy, and Fredrickson equation (LDL=TC-HDL-TG/0.5) [14]. VLDL levels were measured based on the relationship of (VLDL=TG/5). The fasting blood glucose (FBG) rate was obtained with a modified hexokinase enzymatic method 7020 clinical analyzers (Hitachi, Japan). The insulin resistance is determined by the following formula [19]: HOMA-IR (Homeostasis assessment model $)=[$ fasting insulin $(\mu \mathrm{U} / \mathrm{ml})] \times[$ fasting glucose (mmol/l)]\}/22.5. Fasting blood glucose measured by Pars Azmoon kit (Pars Azmoon, Iran) and fasting insulin and serum FGF-21 evaluated by Elecsys Uno Full ELISA device (Crystal Day Biotech co., China).

The $\mathrm{VO}_{2}$ max test results were entered into the Fitness Gram software ver. 10 to calculate the $\mathrm{VO}_{2}$ max. The effects of three types of training on measured variables were examined by the ANCOVA test. The normality of data was examined by the KolmogorovSmirnov test, and equality of variances was calculated by Levene's test. Data were analyzed by SPSS 21 software. The significant level was considered $\mathrm{p}<0.05$.

\section{Findings}

Sixty students were the participant, and the mean of their age was $10.05 \pm 2.4$ years. Their $\mathrm{BMI}$ and $\mathrm{VO}_{2}$ max were $32.36 \pm 2.31 \mathrm{~kg} / \mathrm{m}^{2}$ and $23.06 \pm 1.72 \mathrm{lit} / \mathrm{kg} / \mathrm{min}$, respectively, which showed that subjects were nonactive (sedentary) and obese in pre-training. Also, basic characteristics of subjects Such as age, BMI, and $\mathrm{VO}_{2}$ max were not different among the four groups, significantly $(\mathrm{p}<0.05)$.

Results showed that eight weeks of EE, RE, and CE had a significant effect on increasing serum HDL $(\mathrm{F}=3.04, \mathrm{p}<0.001)$ and on decreasing of glucose $(\mathrm{F}=2.98, \mathrm{p}<0.001), \mathrm{TC}(\mathrm{F}=4.33, \mathrm{p}<0.001), \mathrm{TG}(\mathrm{F}=5.44$, $\mathrm{p}<0.001)$, LDL $(\mathrm{F}=4.86, \mathrm{p}<0.001)$, VLDL $(\mathrm{F}=5.58$, $\mathrm{p}<0.001)$. There were significant differences among all groups in time, and between groups and timegroups. Also, results illustrated that the EE method was better than RE and CE and CE was better than the RE method on improving some variables (Table 1).

Table 1) Changes in variables between pre-training and posttraining (Mean \pm SD)

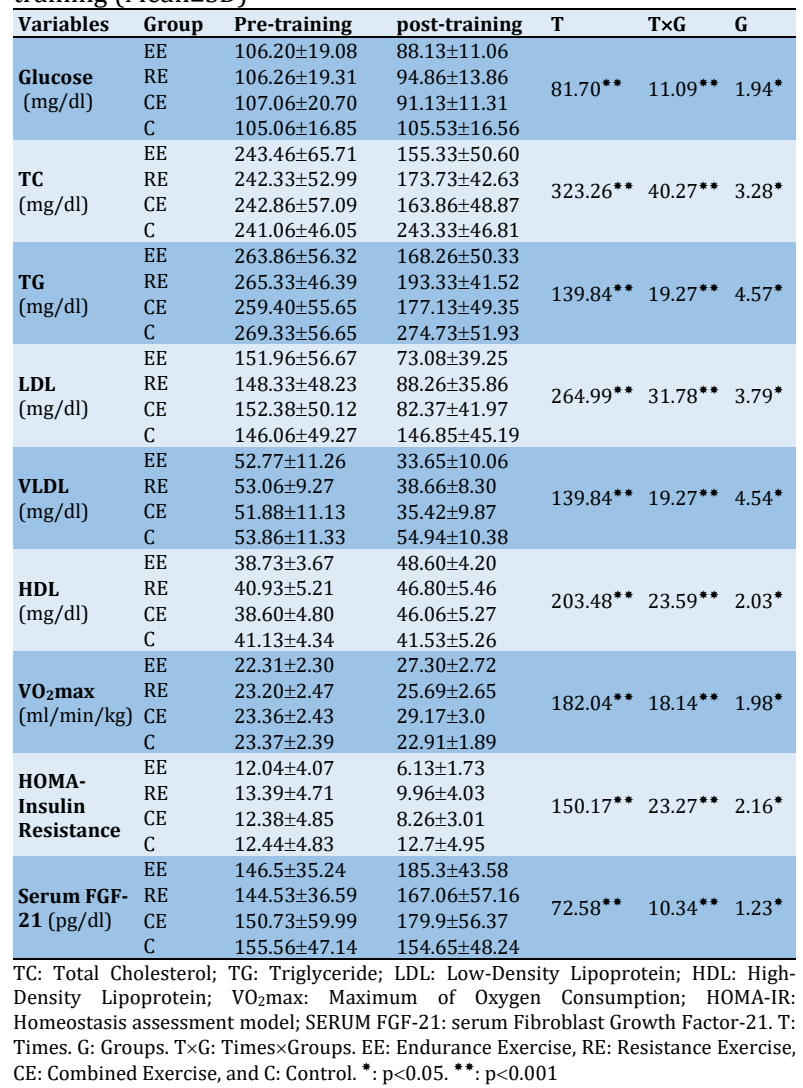

There was a significant difference between the training intervention groups in pre-training and posttraining in the HOMA-Insulin Resistance variable $(\mathrm{F}=3.28, \mathrm{p}<0.001$ ), which had decreased (Diagram 1) and serum FGF-21 ( $\mathrm{F}=2.45, \mathrm{p}<0.001)$, which had increased (Diagram 2). Also, results illustrated that the EE method was better than RE and CE on improving these variables (Diagrams 1 and 2).

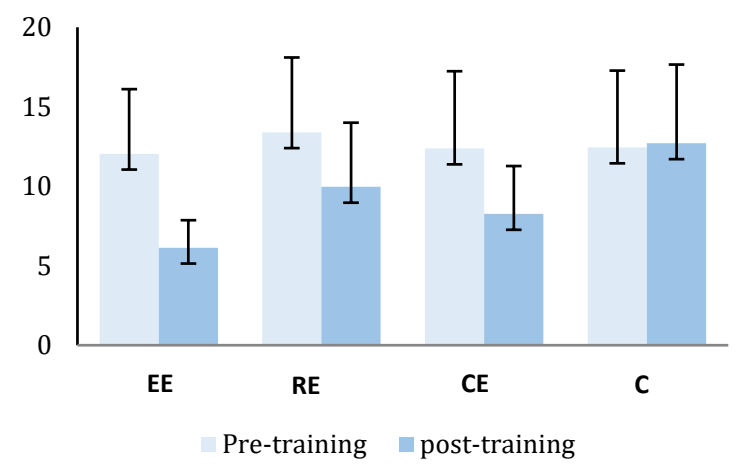

Figure 1) HOMA-Insulin Resistance changes in pre-training and post-training. EE: Endurance Exercise, RE: Resistance Exercise CE: Combined Exercise, and C: Control. 
99

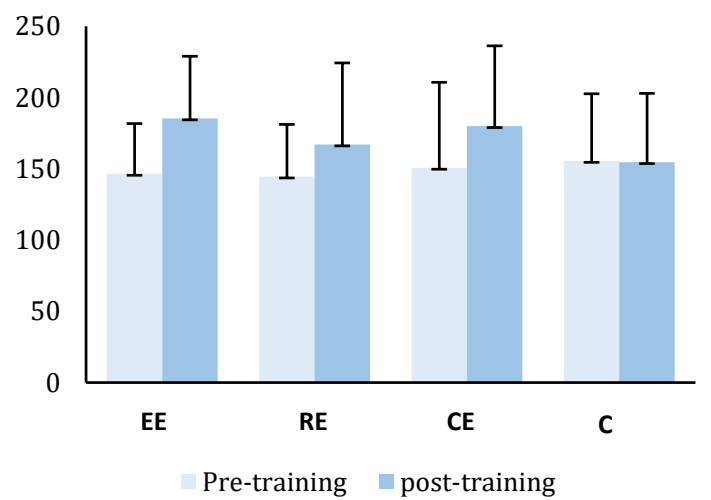

Figure 2) FGF-21 (pg/dl) changes in pre-training and posttraining. EE: Endurance Exercise, RE: Resistance Exercise, CE: Combined Exercise, and C: Control.

\section{Discussion}

This study showed no statistically significant changes in Tc, TG, VLDL, LDL, and HDL levels between groups. However, compared to the control group, these changes had a significant difference in training groups. These findings align with previous studies [4, 12,13,15], and some studies found conflicting results [14]. It seems that many cardiovascular diseases are the result of obesity and related abnormalities in childhood [20-24]. In this regard, Taghian and colleagues have examined the effects of aerobic training on blood lipid levels in obese girls [5]. The above research results showed that serum LDL and TG decreased significantly after three months of endurance training in the training group, but no significant increase was observed in serum HDL [5]. Researchers suggested that this might happen due to the inadequacy of home exercise intensity in the above study. While in the present research, HDL increased in training groups after interventions significantly [15]. In another study, researchers have shown that regular exercise can reduce the levels of blood lipids such as lowering LDL, TG, TC, and increased VLDL and HDL, which can reduce the formation of atherosclerotic plaques [4]. Many studies have shown that practice can reduce body fat mass and fat mass because it is local to the secretion of cardiovascular risk factors and blood lipid profiles [13]. Another study was conducted by Kim and his colleagues in which they examined the effect of 12 weeks of regular endurance training on blood lipid profile values, insulin resistance, and serum adiponectin in adolescent students with a body mass

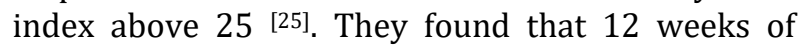
regular endurance training performed five times per week and each session lasting 50 minutes reduced the amounts of fat in their blood profiles [15]. Endurance training increases the use of fat reserves due to the increased density of mitochondria, oxidative enzyme capacity increases in the muscles. In addition, the increased activity of the electron transport chain enzymes involved in the oxidation of fats increases the activity of enzymes in oxidation, especially those involved in the activity of lipoprotein lipase [5].

This research showed no significant difference in changes in insulin resistance between the EE group compared to the CE group and between the CE group compared to the RE group. However, results presented a significant difference in insulin resistance changes between training groups compared to the control group. Some studies [26-34] have reached similar conclusions to the present study, and other research findings were opposite [1]. Regular physical exercise could improve glucose metabolism and insulin resistance [33-35]. Hamedinia and colleagues examined the effects of aquatic exercise (endurance exercise) on insulin resistance in obese children and adolescents [1]. Training groups participated in a training in water $\mathrm{d}$ for eight weeks, three sessions per week, and each session up to 60 minutes. The aquatic exercise showed no significant effect on insulin resistance in children and adolescents. The researchers concluded that eight weeks of endurance training in the water, probably due to less weight change, did not affect insulin resistance in children and adolescents [1]. However, although no significant difference was found in insulin resistance between exercise intervention groups, these exercises improved insulin resistance in this study. This difference can be due to the type of exercise intervention. The available evidence suggests that mechanisms of RET on glucose metabolism and insulin action are similar to the effect of EET. These mechanisms include improving the speed of glucose uptake, increased glucose transporter protein in muscle, increased gene expression or activity of various proteins involved in the insulin signaling cascade (GLUT4), Increased capillary density, increase muscle mass, and glycogen storage capacity increase is due to elevated activity of glycogen synthesis enzyme [29, 34]. It is known that muscle contraction has effects like insulin on glucose uptake in skeletal muscle protein isolate, and skeletal muscle is the main place of glucose uptake in normal mode. Therefore, it is wise to assume that increasing muscle mass is an effective method for improving insulin sensitivity. In addition, it has been shown that fatty deposits in the muscle triglyceride levels are an important aspect of body combined exercise training and are associated with insulin resistance. Therefore, changes in fat mass inside the muscle may affect insulin sensitivity [29, 35-38].

Regarding the Serum FGF21 changes, there was no significant difference between different training groups. While in the case of Serum FGF21 in training groups compared to the control group, a significant difference was observed. Some studies have reached similar conclusions to the present study $[12,16]$. It has recently been found that in young men with normal body mass index, after only 2 weeks of endurance training 5 days a week of Bruce protocol on treadmill exercise performed by participants, FGF21 increased. 
However, the relationship between physical activities and FGF21 is not clear yet. ${ }^{[36]}$ Taniguchi and colleagues reported that endurance exercises in fully trained athletes could increase the FGF21 in young men 19 to 29 years more than those who do not exercise. This means that endurance exercise can improve FGF21 conditions [16]. FGF21 has an important role in the metabolism of fats and carbohydrates and energy balance. FGF21 can increase lipolysis through the interaction of beta- 3 adrenergic receptors on epinephrine in adipose tissue. It is also capable of decreasing blood glucose levels through the action of insulin. This function is performed through the AMPK signaling pathway and causes the transfer of GLUT4 to the cell membrane and muscle glucose uptake. Still, its performance in humans has not been fully understood in physiological conditions [18]. FGF21 has been shown to increase catecholamine and decrease serum insulin, so it increases lipolysis and metabolic changes [12]. As has been said above, FGF21 can affect lipid metabolism, lipid profile, glucose, insulin (insulin resistance).

The limitations that exist in this research can be classified into two parts, including the researcher's uncontrollable limitations and the researcher's controllable limitations, which are stated below. Limitations that have not been studied in control: hereditary characteristics and genetic structures of the subjects and the resting metabolism of the subjects. Limitations that are under the control of the researcher: intensity, volume, and time of training by applying the principles of training, especially endurance, resistance, and combination exercises and its implementation in the training session to test the location, conditions, and safety of training equipment, non-use Medication by subjects by eliminating patient subjects who were taking a particular drug and the amount and type of exercise and daily activity of the subjects.

Based on the information obtained in this study, it can be suggested that if it is found that the cause of the disease in children is obesity, under the supervision of medical and sports experts, such exercises should be used for prevention or possibly for treatment. Also, the Ministry of Education can improve the health status of obese children by using the exercise training used in the present study. Besides, the Ministry of Sports and Youth can implement special training programs similar to this research to promote public sports and improve public health in the country, especially for obese children. The authors suggest to other researchers that in future research, perform the effect of endurance, resistance, and combination exercises for more than eight weeks.

\section{Conclusion}

Endurance training, resistance training, and combined training improve cardiovascular risk factors associated with obesity. These types of training methods also improve cardiovascular risk factors in school obese children. Also, they can be used as effective exercise programs for these people. Therefore, EET, RET, and CET used in this study, especially EET, can be recommended as a nonmedical way to improve the incidence of cardiovascular risk factors and obesity-related disorders in obese boys.

Acknowledgments: We would like to thank the laboratory experts for a job well done.

Ethical Permissions: This study was approved by the Ethics Committee of Yasuj University of Medical Sciences with the certificate No: IR.YUMS.REC.1394.54.

Conflict of Interests: We declare that they have no conflict of interest in this research work.

Authors' Contributions: Mohammadi Mohammadi J. (First author), Introduction author/Methodologist/Original researcher/Discussion author (26\%); Ramezani A. (Second author), Methodologist/Original researcher/Statistical analyst/Discussion author (24\%); Hosseini M. (Third author), Introduction author/Methodologist/Assistant research/Discussion author (20\%); Mohammadi B. (Forth author), Introduction author/Methodologist/Assistant researcher Statistical analyst/Discussion author (20\%); Gaeini B. (Fifth author), Assistant researcher/Statistical analyst/Discussion author (10\%).

Funding/Sources: Funding for this project was provided by Yasuj University of Medical Sciences, Iran.

\section{References}

1- Hamedinia M, Haghighi AH, Sardar MA, PoorJahad J. Effect of training in water on serum adiponectin and insulin resistance in obese children and adolescents. Metab Exerc. 2012;2(2):125-33. [Persian]

2- Lloyd-Jones D, Adams R, Carnethon M, De Simone G, Ferguson TB, Flegal $K$, et al. Heart disease and stroke statistics-2009 update: A report from the American heart association statistics committee and stroke statistics subcommittee. Circulation. 2009;119(3):21-181.

3- Sampasa-Kanyinga H, Standage M, Tremblay MS, Katzmarzyk PT, Hu G, Kuriyan R, et al. Associations between meeting combinations of 24-h movement guidelines and health-related quality of life in children from 12 countries. Public Health. 2017;153:16-24.

4- Siah koohyan M. Effects of endurance training on apoB.apoA1 and LDL size in middle-aged men [dissertation]. Tehran: Tarbiat Modares University; 2001. [Persian]

5- Taghian F, Esfarjani F, Mirsafaei R. The relationship between leptin level changes with some cardiovascular risk factors in obese girls in aerobic training effect. Exerc Physiol. 2010;26:73-85. [Persian]

6- Fowkes FGR, Murray GD, Butcher I, Heald CL, Lee RJ, Chambless LE, et al. Ankle brachial index combined with Framingham risk score to predict cardiovascular events and mortality: A meta-analysis. JAMA. 2008;300(2):197208.

7- Atashak S, Jafari A, Azarbaijani MA. The long-term effects of resistance training on adiponectin and lipid profile in obese men. Razi J Med Sci. 2011;18(86):1-11. [Persian] 8- Bijeh N, Abbasian S. Comparison of the effect of Ramazan month's fasting and regular endurance training on 
101

indicators of lipocalin-2, lipid profile and insulin resistance in inactive obese individuals. Razi J Med Sci. 2013;20(111):16-29. [Persian]

9- Sypniewska G. Laboratory assessment of cardiometabolic risk in overweight and obese children. Clin biochem. 2015;48(6):370-6.

10- Karimi Zarchi AA, Naghiee MR. The prevalence of coronary artery risk factors disease and the effect of lifestyle modification advices. Kowsar Med J. 2009;14(3):157-62. [Persian]

11- Besse-Patin A, Montastier E, Vinel C, Castan-Laurell I, Louche $\mathrm{K}$, Dray $\mathrm{C}$, et al. effect of endurance training on skeletal muscle myokine expression in obese men: Identification of apelin as a novel myokine. Int J Obes. 2014;38(5):707-13.

12- Kim KH, Kim SH, Min YK, Yang HM, Lee JB, Lee MS. Acute exercise induces FGF21 expression in mice and in healthy humans. PLoS One. 2013;8(5):63517.

13- Ramezani AR, Gaeini AA, Hosseini M, Mohammadi J. Effect of endurance, strength and combined training on lipid profile, insulin resistance, and serum adiponectin levels in inactive obese children. ARMAGHANE DANESH. 2016;21(7):641-54. [Persian]

14- Mediano MFF, Neves FA, De Souza Rodrigues Cunha AC, De Souza EPG, Moura AS, Sichieri R. Changes in body weight, C-reactive protein, and total adiponectin in nonobese women after 12 months of a small-volume, homebased exercise program. Clinics. 2013;68(8):1121-7.

15- Ko BJ, Kim SM, Park KH, Park HS, Mantzoros CS. Levels of circulating selenoprotein $\mathrm{P}$, fibroblast growth factor (FGF) 21 and FGF23 in relation to the metabolic syndrome in young children. Int J Obes. 2014;38(12):1497-502.

16- Taniguchi H, Tanisawa K, Sun X, Cao ZB, Oshima S, Ise $\mathrm{R}$, et al. Cardiorespiratory fitness and visceral fat are key determinants of serum FGF21 concentration in Japanese men. J Clin Endocrinol Metab. 2014;99(10):1877-84.

17- Nazem MR, Imami SA, Yaghmaei B, Shekarriz R, Hedaiati M. Serum adiponectin levels in people with low and subclinical hyperthyroid. Iran J Endocrinol Metab. 2013;15(3):279-84. [Persian]

18- Ahmadizad S, Haghighi AH, Hamedinia MR. Resistance versus endurance training on serum adiponectin and insulin resistance index in healthy men. Eur J Endocrinol. 2007;157(5):625-31.

19- Takhshid MA, Qhasemi M. Laboratory method for determining the sensitivity and insulin resistance. J Lab Diagn. 2014;6(23):8-13. [Persian]

20- Ten Hoor GA, Plasqui G, Ruiter RAC, Kremers SPJ, Rutten GM, Schols AMW, et al. A new direction in psychology and health: Resistance exercise training for obese children and adolescents. Psychol Health. 2016;31(1):1-8.

21- Mogharnasi M, Eslami R, Behnam B. Effects of endurance and circuit resistance trainings on lipid profile, heart rate, and hematological parameters in obese male students. Ann Appl Sport Sci. 2014;2(4):11-22. [Persian] 22- Ramezani AR, Gaeini AA, Hosseini M, Mohammadi J. Survey on metabolic cardiovascular risk factors changes following eight weeks of endurance, strength and combined training in sedentary obese children 8-12 years old. Sport Physiol. 2018;9(36):89-108. [Persian]

23- Santos DA, Marques A, Minderico CS, Ekelund U, Sardinha LB. A cross-sectional and prospective analyse of reallocating sedentary time to physical activity on children's cardiorespiratory fitness. J Sports Sci. 2018;36(15):1720-6.
Mohammadi J. et al.

24- Cavarretta E, Mastroiacovo G, Lupieri A, Frati G, Peruzzi $M$. The positive effects of exercise in chemotherapy-related cardiomyopathy. Adv Exp Med Biol. 2017;1000:103-29.

25- Kim JY, Kim ES, Jeon JY, Jekal Y. Improved Insulin resistance, adiponectin and liver enzymes without change in plasma vaspin level after 12 weeks of exercise training among obese male adolescents. Korean J Obes. 2011;20(3):138-46.

26- Thompson PD, Arena R, Riebe D, Pescatello LS. ACSM's new preparticipation health screening recommendations from ACSM's guidelines for exercise testing and prescription, ninth edition. Curr Sports Med Rep. 2013;12(4):215-7.

27- Tanaka H, Monahan KD, Seals DR. Age-predicted maximal heart rate revisited. J Am Coll Cardiol. 2001;37(1):153-6.

28- Ramezani A, Gaeini AA, Hosseini M, Mohammadi J, Mohammadi B. Effects of three methods of exercise training on cardiovascular risk factors in obese boys. Iran J Pediatr. 2017;27(5):7145. [Persian]

29- Molinari-Buchi B, Barth J, Janner M, Frey P. Overweight and obesity in children: Known facts and new trends. Rev Med Suisse. 2010;6(249):1022-5.

30- Abramson JL, Vaccarino V. Relationship between physical activity and inflammation among apparently healthy middle-aged and older U.S. adults. Arch Intern Med. 2002;162(11):1286-92.

31- De Boer RA, Nayor M, De Filippi CR, Enserro D, Bhambhani V, Kizer JR, et al. Association of cardiovascular biomarkers with incident heart failure with preserved and reduced ejection fraction. JAMA Cardiol. 2018;3(3):215-24.

32- Williams MA, Haskell WL, Ades PA, Amsterdam FA, Bittner V, Franklin BA, et al. Resistance exercise in individuals with and without cardiovascular disease: 2007 update: A scientific statement from the American heart association council on clinical cardiology and council on nutrition, physical activity, and metabolism. Circulation. 2007;116(5):572-84.

33- Kriketos AD, Gan SK, Poynten AM, Furler SM, Chisholm DJ, Campbell LV. Exercise increase adiponectin levels and insulin sensivity in humans. Diabetes Care. 2004;27(2):629-30.

34- Holten MK, Zacho M, Gaster M, Juel C, Wojtaszewski JFP, Dela F. Strength training increases in insulin mediated glucose uptake, GLUT4, content, and insulin signaling in skeletal muscle in patients with type 2 diabetes. Diabetes. 2004;53(2):294-305.

35- Amouzou C, Breuker C, Fabre O, Bourret A, Lambert K, Birot 0 , et al. Skeletal muscle insulin resistance and absence of inflammation characterize insulin-resistant grade I obese women. PLoS One. 2016;11(4):0154119.

36- Justesen JM, Andersson EA, Allin KH, Sandholt CH, Jorgensen $\mathrm{T}$, Linneberg $\mathrm{A}$, et al. Increasing insulin resistance accentuates the effect of triglyceride-associated loci on serum triglycerides during 5 years. J Lipid Res. 2016;57(12):2193-99.

37- Srisawat K, Shepherd SO, Lisboa PJ, Burniston JG. A systematic review and meta-analysis of proteomics literature on the response of human skeletal muscle to obesity/type 2 diabetes mellitus (T2DM) versus exercise training. Proteomes. 2017;5(4):30.

38- Yang SJ, Hong HC, Choi HY, Yoo HJ, Cho GJ, Hwang TG, et al. Effects of a three-month combined exercise programme on FGF21 and fetuin-a levels and arterial stiffness in obese women. Clin Endocrinol. 2011;75(4):464-9. 